\title{
Correspondence
}

\section{Epiglottic hematoma: a rare occurrence after tracheal intubation}

To the Editor:

Compromise of the upper airway associated with tracheal intubation often involves trauma to the teeth or gums. ${ }^{1}$ Less frequently, other anatomical structures are involved. We report a case of an epiglottic hematoma following an uneventful general anesthetic. Consent for the use of personal health information was obtained in accordance with our local institutional guidelines.

A 75-yr-old female patient presented for elective left inguinal hernia repair. Her medical history included paroxysmal atrial fibrillation (AF) and sick sinus syndrome. A pacemaker was inserted one week prior to her surgery. She had previous surgeries under general anesthesia without complication. Her medications included warfarin $3.5 \mathrm{mg}$ po qd for $\mathrm{AF}$ and celecoxib 200 po mg qd for arthritic pain. The former was discontinued ten days and the latter two days prior to her operation. Other medications included sotalol 40 mg po bid and transdermal fentanyl $25 \mu \mathrm{g}$ every three days. Her preoperative platelet count, prothrombin time (PT), and activated partial thromboplastin time (APTT) were in the normal range. The procedure was done under general anesthesia. Following induction of anesthesia, a $7.5-\mathrm{mm}$ endotracheal tube was inserted into the trachea without difficulty (Cormack Lehane grade I view). Surgery was uneventful. At the end of the procedure the oropharynx was suctioned prior to tracheal extubation, and the patient was transferred to the recovery room. Several hours later the patient complained of sore throat and hoarseness. This was attributed to the tracheal intubation, and she was discharged home with reassurance that the symptoms would resolve. However, five days later she presented to the emergency room with worsening sore throat, hoarseness and dysphagia. There were no clinical signs of upper airway obstruction. Notably, she had not yet resumed warfarin or celecoxib. The $x$-ray and computed tomography scan showed an enlarged epiglottis with normal subglottic anatomy (Figures 1,2). Fibreoptic examination of the upper airway revealed a large epiglottic hematoma. The patient was subsequently admitted to the intensive care unit and

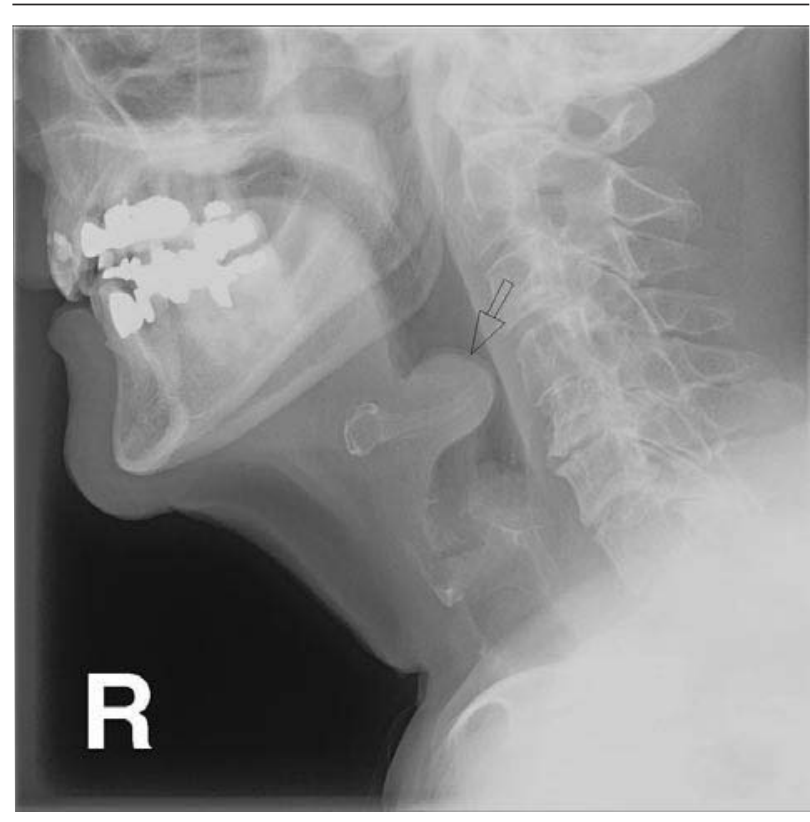

FIGURE $1 \quad x$-ray of the neck shows an enlarged epiglottis measuring $2.3 \mathrm{~cm}$ in vertical dimension and $2.5 \mathrm{~cm}$ in antero-posterior dimension (lateral view).

treated for three days with dexamethasone $8 \mathrm{mg}$ in $\mathrm{q} 8 \mathrm{~h}$ (no supplemental oxygen was administrated, the patient remained on R/A). Fibreoptic examination three days later showed a decrease in epiglottic size, and the patient was discharged the following day. She has remained symptom free since.

Epiglottic hematoma associated with endotracheal intubation is rare. One previous report described an epiglottic hematoma, recognized following two attempts at endotracheal intubation. ${ }^{2}$ The hematoma was evacuated immediately, and there were no postoperative sequelae. In a second report ${ }^{3}$ an epiglottic hematoma developed in a patient who was being treated with warfarin. Intubation of his trachea was uneventful. However, in the recovery room the patient experienced sore throat, hemoptysis, and dysphagia. He required emergency tracheotomy for upper airway obstruction. In contrast to these cases, the epiglottic hematoma we describe was not diagnosed until five days postoperatively. Although the patient did not 


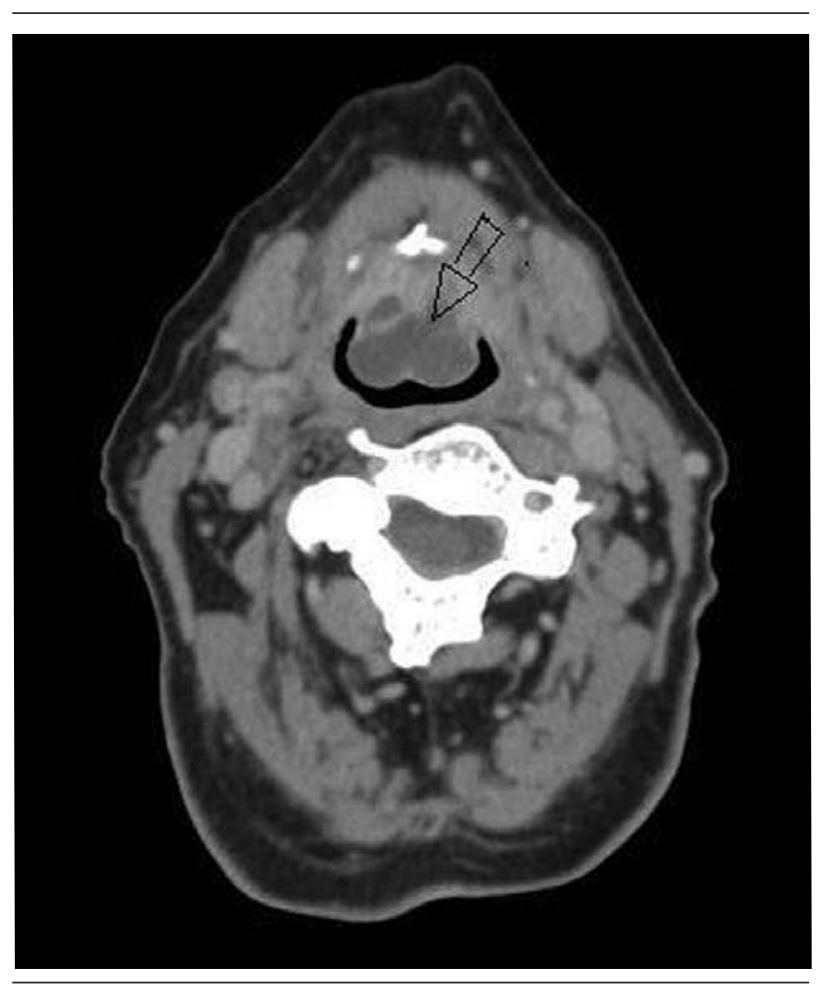

FIGURE 2 Computed tomography scan of the neck reveals an enlarged epiglottis (cross section view).

demonstrate signs of upper airway obstruction during the initial postoperative period, she complained of sore throat, hoarseness and dysphagia. Trauma to the epiglottis could have occurred during intubation or extubation of the trachea, possibly in association with suctioning of the oropharynx. Anticoagulant medications have also been identified as a possible risk factor in association with epiglottic hematoma. ${ }^{3,4}$ This was unlikely to have been an issue in this case, as the patient's warfarin had been discontinued, and she had normal PT and APTT values preoperatively. It is uncertain if a residual anti-platelet effect of her nonsteroidal anti-inflammatory drug (discontinued two days prior to surgery) may have been an associated risk factor in development of an epiglottic hematoma.

This report highlights that sore throat, hoarseness and dysphagia following endotracheal intubation could be presenting symptoms of the rare, but potentially serious complication of an epiglottic hematoma. This entity should be considered in the differential diagnosis of upper airway lesions when confronted with this triad of symptoms following anesthesia where endotracheal intubation was performed, especially in patients receiving concurrent anticoagulation therapy.
Roupen Hatzakorzian MD FRCPC

William Li Pi Shan MD FRCPC

Steven B. Backman MDCM PHD FRCPC

Royal Victoria Hospital, Montreal, Canada

E-mail: roupenhatz@hotmail.com

Accepted for publication December 21, 2005.

\section{References}

1 Owen H, Waddell-Smith. Dental trauma associated with anaesthesia. Anaesth Intensive Care 2000; 28: 133-45.

2 Cheng KS, Li HY. Epiglottic hematoma secondary to endotracheal intubation. Acta Anaesthesiol Sin 1999; 37: 49-52.

3 Brown I, Kleinman B. Epiglottic hematoma leading to airway obstruction after general anesthesia. J Clin Anesth 2002; 14: 34-5.

4 Lee M, Berger HW, Granada MG. Acute upper airway obstruction. Sodium warfarin-induced hemorrhage into the base of the tongue and epiglottis. Chest 1980; 77: 454-5.

\section{Morbidity in a pediatric patient having alcobol ablation of an arteriovenous malformation}

To the Editor:

An otherwise healthy 25 -month-old, $11.8 \mathrm{~kg}$ male child was scheduled for alcohol embolization of an arteriovenous malformation on the back of his skull. The procedure was performed under general anesthesia. The duration of the case was approximately three hours and $45 \mathrm{~min}$, and the patient remained hemodynamically stable throughout. After obtaining a cerebral angiogram, the arteriovenous malformation and fistula were treated with $11.5 \mathrm{~mL}$ of anhydrous alcohol administered in 2-3 mL increments over approximately one hour. The procedure was stopped after a dose of $1 \mathrm{~mL} \cdot \mathrm{kg}^{-1}$ of alcohol had been injected.

In order to monitor the metabolic effects of the injected alcohol, arterial blood gas chemistry and serum ethanol levels were obtained at the conclusion of the case. His arterial $\mathrm{pH}$ was $7.19, \mathrm{PaCO}_{2} 50$ $\mathrm{mmHg}, \mathrm{Pa}_{2} 121 \mathrm{mmHg}, \mathrm{HCO}_{3}^{-} 19 \mathrm{mmol} \cdot \mathrm{L}^{-1}, \mathrm{BE}-$ 9 , anion gap $31, \mathrm{Na}^{+} 133 \mathrm{mmol} \cdot \mathrm{L}^{-1}, \mathrm{~K}^{+} 4.6 \mathrm{mmol} \cdot \mathrm{L}^{-1}$, HCT $26 \%$ (preoperative $31.9 \%$ ). The urine was serosanguinous at this time, and the serum alcohol level was $85.2 \mathrm{mg} \cdot \mathrm{dL}^{-1}$ (legal intoxication is defined as > $\left.80 \mathrm{mg} \cdot \mathrm{dL}^{-1}\right)$.

In response to the metabolic derangement, the patient received sodium bicarbonate $15 \mathrm{mEq} i$. He was allowed to emerge from anesthesia, and his trachea was extubated in the catheterization labora- 